\title{
Characterization of deep levels in semi-insulating gallium arsenide
}

\author{
Y N MOHAPATRA, N BALASUBRAMANYAM and \\ VIKRAM KUMAR \\ Department of Physics and Materials Research Laboratory, Indian Institute of Science, \\ Bangalore 560012, India \\ MS received 27 February 1984; revised 26 December 1984
}

\begin{abstract}
Two traps with activation energies of $E_{c}-0.47 \mathrm{eV}$ and $E_{v}+0.79 \mathrm{eV}$ have been detected in semi-insulating $\mathrm{GaAs}$ : $\mathrm{Cr}$ through optical transient current spectroscopy (OTCS) in the temperature range $300-450 \mathrm{~K}$. The latter trap gives rise to rising current transients which result in a negative peak in the oTCS spectrum. The theoretical expressions for current transients have been derived.
\end{abstract}

Keywords. Gallium arsenide; deep levels; activation energy; current transients; optical transient current spectroscopy.

\section{Introduction}

Semi-insulating gallium arsenide is of great technological importance due to its application as substrates for microwave devices. Hence chromium-doped semiinsulating gallium arsenide has been widely studied; yet the understanding of the trap levels responsible for the characteristic properties of this material remains poor. This is mainly because popular methods of electrical characterization such as DLTS cannot be used. Most of the measurements on GaAs: $\mathrm{Cr}$ have been performed on semiconducting samples. Martin and Bois (1978) introduced optical transient current spectroscopy (отсs) to facilitate study of deep levels in semi-insulating samples. In this technique, a pulse of intrinsic light is used to fill the traps, and the transient conductivity due to emission of carriers from the traps on removal of light is analysed as in DLTs. However there have been difficulties in interpreting the results. Deveaud and Toulouse (1980) used this technique but had to invoke a large number of free parameters to interpret their experimental data, particularly the negative peaks. They have thus concluded that semi-insulating GaAs has a large number of trap levels.

In this paper we describe the results of our otcs experiments on semi-insulating GaAs: $\mathrm{Cr}$ which lend themselves to simple interpretation. In $\S 2$, we derive the expressions for the current transient and give experimental details in $\S 3$. We discuss our results in the light of the already published data in $\S 4$.

\section{Theory}

Here we consider the electron occupancy of deep levels in the band gap during and after a pulse of intrinsic light. The rate of change of electron occupied trap concentration is given by (Sah et al 1970) 


$$
\frac{\mathrm{d} n_{T}}{\mathrm{~d} t}=-\left(c_{n} n+e_{n}+c_{p} p+e_{p}\right) n_{T}+\left(c_{n} n+e_{p}\right) N_{T}
$$

where $N_{T}$ is the total trap concentration and all other symbols have their usual meanings. The emission terms are sums of the respective thermal and optical contributions.

When high intensity intrinsic light is allowed to fall on the sample, a large number of electron-hole pairs are created changing the occupancy of the trap levels. On reaching the steady state with light on, the trap occupancy

$$
\frac{n_{T}}{N_{T}}=\frac{c_{n} n+e_{p}}{c_{n} n+e_{n}+c_{p} p+e_{p}} \simeq \frac{c_{n}}{c_{n}+c_{p}}
$$

where the second equality is obtained by neglecting the emission terms and assuming $n=p$ during this period. When the light is turned off at $t=0$, mainly two processes occur: (i) the carriers in the bands recombine rapidly with a time constant $\tau_{r}$, (ii) the traps emit carriers slowly resulting in a transient current. The concentration of the occupied trap is governed by the rate equation.

$$
\frac{\mathrm{d} n_{T}}{\mathrm{~d} t}=-\left(e_{n}+e_{p}\right) n_{T}+e_{p} N_{T}
$$

as the capture processes are negligible and has the solution

$$
n_{T}(t)=\left[n_{T}(0)-n_{T}(\infty)\right] \exp \left[-\left(e_{n}+e_{p}\right) t\right]+n_{T}(\infty),
$$

where the initial condition is given by (2) and the final condition by

$$
\frac{n_{T}(\infty)}{N_{T}}=\frac{e_{p}}{e_{n}+e_{p}}
$$

The concentration of the electrons in the conduction band contributing to the conductivity is determined by

$$
\frac{\mathrm{d} n}{\mathrm{~d} t}=-\frac{n}{\tau_{r}}+e_{n} n_{T}
$$

If $\tau_{r}$ is so short that $\frac{\mathrm{d} n}{\mathrm{~d} t} \ll \frac{n}{\tau_{r}}(\operatorname{Rose} 1963)$,

$$
n=\tau_{r} e_{n} n_{T}
$$

Similarly the hole concentration in valence band

$$
p=\tau_{r} e_{p}\left(N_{T}-n_{T}\right)
$$

Using (7), the time dependent part of the current can be written as

$$
i(t)=A \sigma(t) \varepsilon=A \tau_{r} q \varepsilon\left(\mu_{n} e_{n}-\mu_{p} e_{p}\right) n_{T}(t)
$$

where $A$ is the area and $\varepsilon$ the applied field. Using (2), (4) and (5)

$$
i(t)=A \tau_{r} q \varepsilon N_{T}\left(\mu_{n} e_{n}-\mu_{p} e_{p}\right)\left[\frac{c_{n}}{c_{n}+c_{p}}-\frac{e_{p}}{e_{n}+e_{p}}\right] \exp \left[-t\left(e_{n}+e_{p}\right)\right] .
$$

The current transient would be decaying type if $e_{n} \gg e_{p}$ and $c_{n} \gg c_{p}$ i.e. a trap in the 
upper half of the band gap communicating with the conduction band, since ( 9 ) becomes

$$
i(t)=A q \tau_{r} \varepsilon N_{T} \mu_{n} e_{n} \exp \left(-e_{n} t\right) .
$$

On the other hand, a rising current transient can also occur if $\mu_{p} e_{p}<\mu_{n} e_{n}$ and

$$
\frac{c_{n}}{c_{n}+c_{p}}<\frac{e_{p}}{e_{n}+e_{p}}
$$

Thus a trap in the upper half of the bandgap with hole capture cross-section larger than the electron capture cross-section will result in a rising current transient and hence a negative orcs peak. Analogous conditions prevail for traps in the lower half of the bandgap.

\section{Experiment}

Electroless gold films are deposited on both surfaces of chromium doped semiinsulating GaAs wafers (Wacker Chemitronic). The front surface is mesa-etched to allow light penetration. The chopped output of a $1 \mathrm{~mW}$ He-Ne laser is used as the light source. Current in the sample is measured by a Keithly 427 current amplifier and sampled at times $t_{1}$ and $t_{2}$ after the light is turned off. The orcs signal $S=i\left(t_{1}\right)-i\left(t_{2}\right)$ is directly plotted on an $X-Y$ recorder as a function of sample temperature. A schematic diagram of the experimental set-up is given in figure 1 .

The oTcs signal goes through an extremum (maximum for a decaying transient and minimum for a rising transient) at a temperature at which the emission time constant $\tau$ is given by

$$
\frac{1}{\tau}=\left[1 /\left(t_{2}-t_{1}\right)\right] \ln \left[\left(\tau-t_{2}\right) /\left(\tau-t_{1}\right)\right],
$$

assuming a transient of the type given in (10). The emission time constant is measured as a function of temperature by changing the sampling times $t_{1}$ and $t_{2}$. From detailed

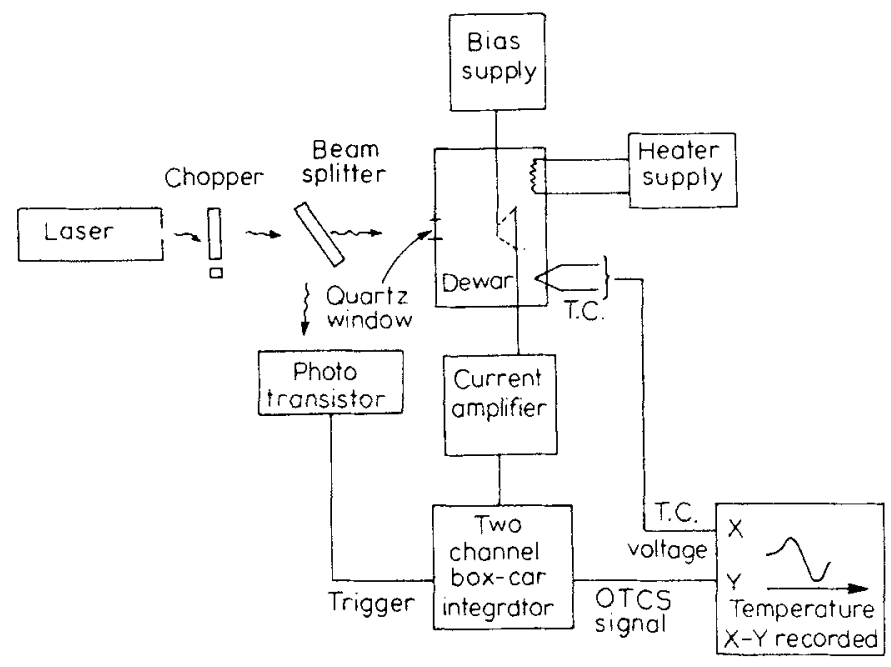

Figure 1. Schematic diagram of the otcs experimental set-up. 
balance, the emission time constant $\tau_{n}$ for electrons is given by

$$
\frac{1}{\tau_{n}}=\sigma_{n}\left\langle V_{t h}\right\rangle_{n} N_{c} \exp (-\Delta E / k T)
$$

where $\sigma_{n}$ is the electron capture cross-section and $\Delta E$ the activation energy and all other symbols have their usual meanings. Hence the slope and $y$-intercept yields the activation energy and capture cross-section respectively.

\section{Results and discussion}

In the temperature range $300-450 \mathrm{~K}$, two peaks $A$ and $B$ are observed in the oTcs spectrum of which one is positive and other negative (figure 2). The peak temperature of level $A$ does not correspond to the true time constant given by (12) since it is affected by the much stronger $B$ peak. Therefore, full isothermal optical transient currents are recorded to determine the time constant for this level at different temperatures. The Arrhenius plot shown in figure 3 yields an activation energy of $0.47 \mathrm{eV}$ with capture cross-section $\sigma_{n}=1 \times 10^{-17} \mathrm{~cm}^{2}$ from the detailed balance relation, (13).

The activation energy of the negative peak obtained from $\ln \left(\tau T^{2}\right)$ vs $1 / T$ plot extending over three decades is $0.79 \mathrm{eV}$. The corresponding capture cross-section $\sigma_{p}=3.2 \times 10^{-15} \mathrm{~cm}^{2}$ is obtained from this plot. We believe that this is the trap generally associated with $\mathrm{Cr}$ at the $\mathrm{Ga}$ site. From DLTs and oDlts on conductive GaAs:Cr samples Martin et al (1980) found an energy level at $0.75 \mathrm{eV}$ in bulk, vPE and LPE materials. Szawelska and Allen (1979) have associated hole trap with an ionization energy of $0.81 \mathrm{eV}$ with the transition $\mathrm{Cr}^{3+} \rightarrow \mathrm{Cr}^{2+}$ using photocapacitance measure-

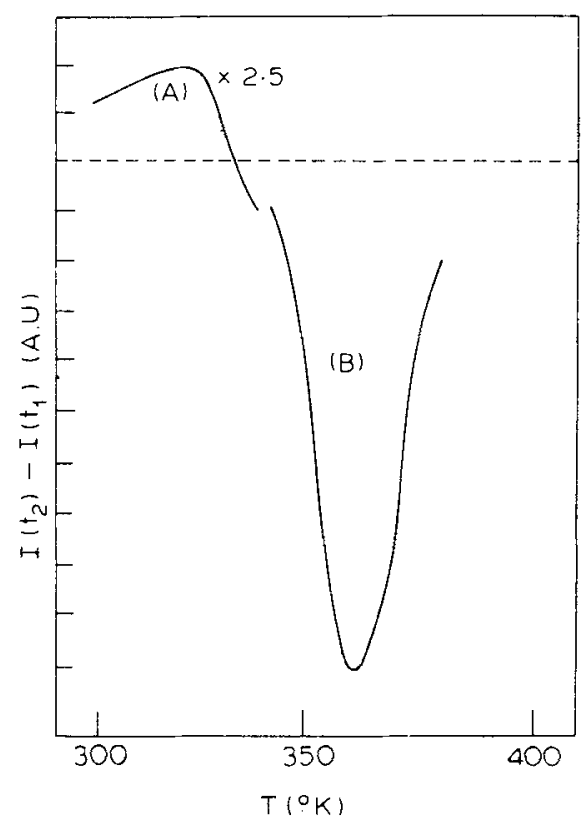

Figure 2. OTCS spectrum of semi-insulating $\mathrm{GaAs}: \mathrm{Cr}$ with $t_{1}=30 \mathrm{msec}$ and $t_{2}=60 \mathrm{msec}$. 


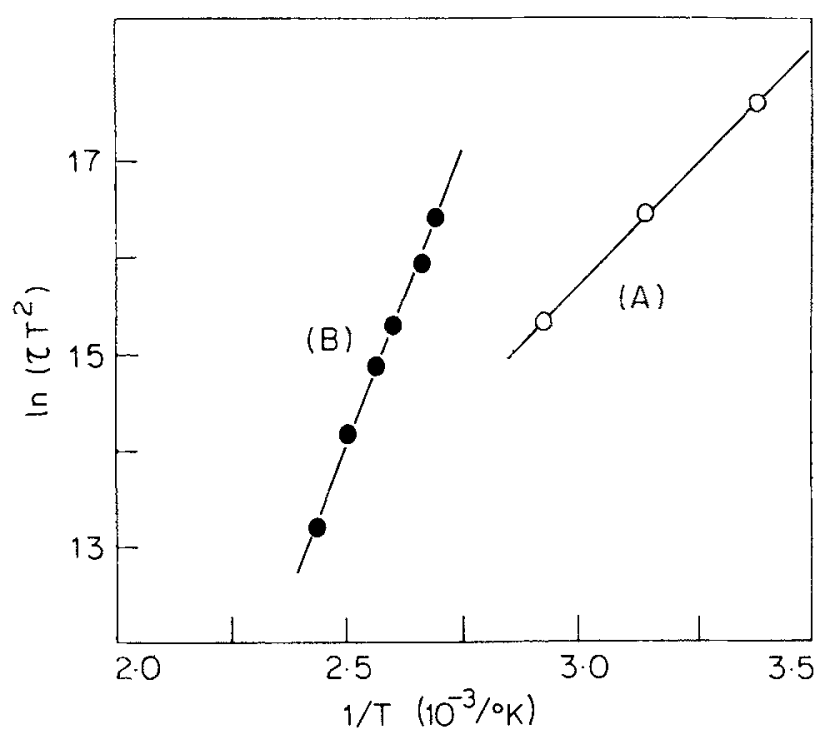

Figare 3. Arrhenius plot for the two levels $A$ and $B$ corresponding to the positive and negative peaks of figure 2 respectively.

ments. Earlier Lang and Logan (1975) have ascribed a trap level at $E_{v}+0.78 \mathrm{eV}$ with $\sigma_{p}=2 \times 10^{-17} \mathrm{~cm}^{2}$ to chromium.

In conclusion, $\mathrm{Cr}$-doped semi-insulating GaAs has two main levels: (i) an electron trap at $E_{\mathrm{c}}-0.47 \mathrm{eV}$ and (ii) a hole trap at $E_{v}+0.79 \mathrm{eV}$.

\section{Acknowledgements}

The authors thank Prof. G Suryan for encouragement. This work was supported by DRDO.

\section{References}

Deveaud B and Toulouse B 1980 Semi-insulating $/ 11-V$ materials (conference), Nottingham (ed,) G J Rees (Kent: Shiva Publishers) pp. 241

Lang D V and Logan R A 1975. J. Electron. Mater. 41053

Martin G M and Bois D 1978 Electrochem. Soc. 7832

Martin G M, Mitonneau A, Pons D, Mircea A and Woodard D W 1980 J. Phys. C13 3855

Rose A 1963 Concepts in photoconductivity and allied problems (New York: John Wiley)

Sah C T, Forbes L, Rosier L L and Tasch A F Jr 1970 Solid State Electron. 13759

Szawelska H R and Allen J W 1979 J. Phys. C12 3359 